\title{
MODELAGEM DA ESTRUTURA DIAMÉTRICA EM ECÓTONO DE FLORESTA OMBRÓFILA MISTA E ESTACIONAL DECIDUAL SUBMETIDA A MANEJO FLORESTAL SUSTENTADO
}

\section{DIAMETER STRUCTURE MODELING IN MIXED TROPICAL AND SEASONAL DECIDUAL ECOTONE FORESTS SUBMITTED TO SUSTAINED FOREST MANAGEMENT}

\author{
Jonathan William Trautenmüller ${ }^{1}$, Sylvio Péllico Netto ${ }^{2}$, Rafaelo Balbinot ${ }^{3}$, \\ Ana Paula Dalla Corte ${ }^{4}$, Rafael Vendruscolo ${ }^{5}$ \\ 1, 2, 4 Universidade Federal do Paraná, Curitiba, Paraná, Brasil -jwtraute@gmail.com, \\ sylviopelliconetto@gmail.com, anapaulacorte@gmail.com \\ ${ }^{3}$ Universidade Federal de Santa Maria, Frederico Westphalen, Rio Grande do Sul, Brasil - \\ rafaelo.balbinot@gmail.com \\ ${ }^{5}$ SALTUS Consultoria Ambiental e Florestal, Frederico Westphalen, Rio Grande do Sul, Brasil - \\ eng.rafaelvendruscolo@gmail.com
}

\begin{abstract}
RESUMO
Este trabalho tem como objetivo avaliar o efeito do manejo florestal em regime sustentado sobre a estrutura diamétrica da comunidade florestal e sobre as quatro espécies com maior frequência, em um fragmento florestal caracterizado como ecótono das florestas Ombrófila Mista e Estacional Decidual. O estudo foi realizado em uma área de 50 ha, localizada no noroeste do estado do Rio Grande do Sul - BR, centrada nas seguintes coordenadas $27^{\circ} 36^{\prime}$ 49,60" Sul e 53²9' 23,05" Oeste. Dois inventários foram realizados, (i) em 1993, no qual foram alocadas 25 parcelas $(20 \mathrm{~m} \times 50 \mathrm{~m}$ ) aleatoriamente, e (ii) em 2013, no qual foram instaladas 31 parcelas $(20 \mathrm{~m} \times 50 \mathrm{~m})$ sistematicamente. Todos as árvores acima de $10 \mathrm{~cm}$ de diâmetro à altura do peito foram mensuradas e identificadas a nível de espécie. Os resultados demonstraram que apenas a função Exponencial $2 \mathrm{P}$ apresentou aderência nos dois anos de mensuração para a comunidade florestal. Para as espécies Cedrela fissilis Vell. e Campomanesia xanthocarpa O. Berg a distribuição SB Johnson apresentou a melhor aderência, já para a Araucaria angustifolia (Bertol.) Kuntze foi a distribuição Beta e para a Sebastiania commersoniana (Baill.) L.B. Sm. e Downs a distribuição LogNormal 3P, considerando os dois inventários. O manejo florestal em regime sustentado apresentou benefícios para algumas espécies como a Campomanesia xanthocarpa e Sebastiania commersoniana, que retomaram suas regenerações em fluxo contínuo, já as espécies Araucaria angustifolia e Cedrela fissilis não apresentaram tal retomada.
\end{abstract}

PALAVRAS-CHAVE: Araucária, Cedro, Distribuições probabilísticas, Plano de manejo.

\begin{abstract}
The aim of this work was to evaluate the effect of sustainable forest management on the diameter structure of forest community and four species with highest frequency in a forest fragment characterized as an ecotone of Mixed Tropical and Seasonal Deciduous forests. The study area has 55 ha and located in the Northwestern of Rio Grande do Sul, BR, centered on the following coordinates: $27^{\circ} 36^{\prime} 49.60^{~ " S}$ and $53^{\circ} 29^{\prime} 23.05^{\prime \prime} \mathrm{W}$. Two inventories were carried out, in 1993, in which 25 plots $(20 \mathrm{~m} \times 50 \mathrm{~m}$ ) were randomly allocated, and in 2013, with 31 plots $(20 \mathrm{~m} \times 50 \mathrm{~m})$ systematically allocated. In which all trees above $10 \mathrm{~cm}$ in diameter at breast height were measured and identified. The results show that the Exponential $2 \mathrm{P}$ distribution was the only one well adjusted (adherent) to the forest community data. For the species Cedrela fissilis and Campomanesia xanthocarpa, SB Johnson distribution presented the best goodness of fit, whereas for Araucaria angustifolia it was the Beta distribution, and for Sebastiania commersoniana the LogNormal 3P distribution. Sustainable forest management has benefits for some species such as Campomanesia xanthocarpa and Sebastiania commersoniana, which returned to their regeneration in a continuous flow, in which the species Araucaria angustifolia and Cedrela fissilis did not present such resumption.
\end{abstract}

KEYWORDS: Araucária, Cedro, Probabilistic distributions, Management plan. 


\section{INTRODUÇÃO}

A Mata Atlântica já foi uma das maiores florestas da América Latina com aproximadamente 150 milhões de hectares (IBGE, 2012). Essa floresta apresenta grande biodiversidade, contendo diversas espécies florestais endêmicas e de alto valor comercial. A exploração descontrolada de madeira e a expansão das fronteiras agrícolas, no passado, acarretaram drástica redução de sua cobertura florestal e, consequentemente, sua fragmentação, restando menos de $2 \%$ da cobertura original (MACHADO et al., 2013). Dentre as diversas tipologias florestais que compõem a Mata Atlântica em áreas subtropicais, destacam-se as florestas Ombrófila Mista e Estacional Decidual. Ainda que essas florestas sejam representadas em sua maioria por fragmentos, torna-se de fundamental importância estudos sobre sua estrutura diamétrica, tanto da comunidade florestal quanto de espécies individuais.

A distribuição diamétrica tornou-se uma ferramenta poderosa para descrever a estrutura de florestas (LOETSCH et al., 1973; TÉO et al., 2015), podendo ser classificadas como unimodal, decrescente e multimodal (LOETSCH et al., 1973; SCOLFORO, 2006; TÉO et al., 2015). Povoamentos florestais equiâneos e sem desbastes geralmente são representados pela distribuição unimodal (BINOTI et al, 2014). As florestas naturais são caracterizadas, em sua maioria, pela forma decrescente conhecida como “Jinvertido" (TÉO et al., 2015; CYSNEIROS et al., 2017). Florestas plantadas equiâneas que sofreram desbastes podem apresentar a distribuição multimodal, porém, para algumas espécies florestais essa distribuição em remanescentes florestais advém devido algum fator perturbante (EBLING \& PÉLLICO NETTO, 2015).

A distribuição diamétrica fornece subsídios para identificar o grau e intensidade da regeneração natural, tanto para espécie, grupos de espécies e para a comunidade florestal (MACHADO et al., 2010; TÉO et al., 2015), constituindo informação importante sobre o estoque da floresta (SCOLFORO, 2006). Esses estudos são de fundamental importância, pois o diâmetro apresenta boa correlação com outras variáveis biométricas. Esses trabalhos podem revelar a dinâmica da regeneração natural (MACHADO et al., 2010; CYSNEIROS et al., 2017), ou até mesmo, as alterações provenientes de manejo florestal em regime sustentado.

O manejo florestal sustentável pode ser considerado um elemento decisivo para perpetuar a sobrevivência de diversas espécies (SILVEIRA et al., 2018), em especial da Araucaria angustifolia (Bertol.) Kuntze, que apresenta dificuldades para manter a regeneração em fluxo contínuo no seu habitat natural, quando não ocorreram distúrbios ou intervenção antrópica (EBLING \& PÉLLICO NETTO, 2015). Manejar tais florestas estimula a volta da regeneração natural, aumenta as taxas de crescimento das árvores remanescentes e reduz suas taxas de mortalidade (HESS et al., 2010; SILVEIRA et al., 2018).

A hipótese proposta é de que a floresta e as espécies em estudo, apresentem distribuição diamétrica exponencial negativa, e que o manejo florestal em regime sustentado influenciou sua estrutura diamétrica. Dessa forma, com o desenvolvimento desse trabalho objetivouse avaliar o efeito do manejo florestal em regime sustentado sobre a estrutura diamétrica da comunidade florestal e das quatro espécies com maior frequência, em fragmento florestal caracterizado como ecótono das florestas Ombrófila Mista e Estacional Decidual.

\section{MATERIAL E MÉTODOS}

O estudo foi realizado em um fragmento florestal caracterizado como transição entre a Floresta Ombrófila Mista e a Floresta Estacional Decidual (IBGE, 2012), com aproximadamente 50 ha. A área está situada em Erval Seco, Rio Grande do Sul, Brasil, centrado nas coordenadas $27^{\circ} 36^{\prime} 49,60^{\prime \prime}$ S e 5329'23,05" W. O clima é subtropical úmido (Cfa) segundo Köppen (ALVARES et al., 2013), com regime de chuvas equilibrado durante o ano todo com precipitação média anual entre 1.900 e $2.200 \mathrm{~mm}$ e temperatura média variando de 18 a $20^{\circ} \mathrm{C}$ (ROSSATO, 2014). Os solos são classificados como Latossolo Vermelho Alumino Férrico (SANTOS et al., 2013).

No ano de 1993 foi realizado inventário florestal em 25 parcelas de $20 \mathrm{~m} \times 50 \mathrm{~m}$ distribuídas aleatoriamente em todo o fragmento, encontrando uma densidade de 439 árvores por hectare e $210,83 \mathrm{~m}^{3} \mathrm{ha}^{-1}$. Após esse inventário foi realizado manejo florestal em regime sustentado, no qual, foi suprimido $55,83 \mathrm{~m}^{3} \mathrm{ha}^{-1}$. Esse volume explorado corresponde as classes de diâmetro acima de $30 \mathrm{~cm}$ de diâmetro à altura do peito (DAP). Em 2013 foi efetuado inventário em 31 parcelas de 20 m x 50 m, distribuídas em um grid sistemático, com equidistância de $125 \mathrm{~m} \times 125 \mathrm{~m}$. Nas parcelas foram mensurados os diâmetros e a altura total, de todas as árvores (DAP $\geq 10 \mathrm{~cm}$ ) nas duas ocasiões.

Foram selecionadas as quatro espécies com maior frequência, somados os dois inventários, sendo elas Araucaria angustifolia (Bertol.) Kuntze, Cedrela fissilis Vell., Campomanesia xanthocarpa O. Berg e Sebastiania commersoniana (Baill.) L.B. Sm. e Downs. Os parâmetros fitissociologicos desses inventários estão disponíveis em 
Vendruscolo (2015).

As funções densidade de probabilidade (fdps) testadas foram: i) Beta, ii) Exponencial 2P, iii) Gama 3P, iv) Log Normal 3P, v) SB Johnson e vi) Weibull 3P, para a obtenção das distribuições diamétricas da floresta e para as quatro espécies selecionadas. Todas as distribuições ajustadas foram avaliadas pelo teste de Kolmogorov-Smirnov, conforme conceitos e aplicações apresentados por Longhi et al. (2017) e pelo erro padrão da estimativa ( $\left.S_{y x} \%\right)$. Com os resultados do ajuste, foi gerada uma classificação em ordem crescente de maior aderência do ajuste. Foi aplicado o teste de qui-quadrado para verificar se houve diferença significativa entre as distribuições de 1993 e 2013.

Para definir o número de classes de diâmetro, utilizouse o método de Sturges (STURGES, 1926). A assimetria e a curtose foram implementadas e avaliadas conforme aplicadas por Machado et al. (2006). Todos os ajustes foram efetuados com o auxílio do programa Easyfit.

\section{RESULTADOS E DISCUSSÃO}

A estatística descritiva evidenciou que para a floresta e para $A$. angustifolia, $C$. fissilis e $S$. commersoniana o diâmetro máximo reduziu passados 20 anos da execução do manejo florestal em regime sustentado. Apenas a $C$. xanthocarpa não apresentou redução do diâmetro máximo (Tabela 1). Destaca-se a redução do diâmetro máximo da C. fissilis de aproximadamente oito centímetros.

Tabela 1. Estatísticas descritivas do diâmetro à altura do peito (DAP) para a floresta e para as espécies de maior frequência na floresta, caracterizada como ecótono das florestas Ombrófila Mista e Estacional Decidual.

\begin{tabular}{|c|c|c|c|c|c|}
\hline \multirow{2}{*}{ Estatísticas } & \multirow{2}{*}{ Floresta } & \multicolumn{4}{|c|}{ Espécies } \\
\hline & & Araucaria angustifolia & Cedrela fissilis & Campomanesia xanthocarpa & Sebastiania commersoniana \\
\hline \multicolumn{6}{|c|}{1993} \\
\hline № & 1098 & 83 & 58 & 96 & 38 \\
\hline Média (cm) & 26,79 & 38,81 & 31,63 & 19,68 & 26,17 \\
\hline Mediana $(\mathrm{cm})$ & 21,96 & 38,83 & 30,39 & 18,78 & 27,21 \\
\hline Moda $(\mathrm{cm})$ & 10,82 & 10,82 & 17,82 & 18,78 & 10,3 \\
\hline $\operatorname{Dmin}(\mathrm{cm})$ & 10,0 & 10,0 & 10,0 & 10,0 & 10,0 \\
\hline $\operatorname{Dmax}(\mathrm{cm})$ & 104,72 & 82,76 & 67,80 & 48,70 & 63,98 \\
\hline Amplitude (cm) & 94,72 & 72,76 & 57,80 & 38,70 & 53,98 \\
\hline Variância $\left(\mathrm{cm}^{2}\right)$ & 233,60 & 393,95 & 265,01 & 53,10 & 174,98 \\
\hline Desvio Padrão (cm) & 15,28 & 19,85 & 16,28 & 7,28 & 13,23 \\
\hline Assimetria & 1,04 & $-0,004$ & 0,41 & 1,26 & 0,61 \\
\hline Curtose & 0,62 & $-1,29$ & $-1,03$ & 2,74 & 0,04 \\
\hline \multicolumn{6}{|c|}{2013} \\
\hline № & 1288 & 85 & 41 & 89 & 67 \\
\hline Média (cm) & 24,15 & 44,21 & 35,99 & 20,70 & 24,77 \\
\hline Mediana $(\mathrm{cm})$ & 19,07 & 45,57 & 37,72 & 17,89 & 23,24 \\
\hline Moda $(\mathrm{cm})$ & 18,46 & 58,57 & 40,52 & 24,83 & 10,34 \\
\hline $\operatorname{Dmin}(\mathrm{cm})$ & 10,0 & 10,41 & 10,31 & 10,0 & 10,03 \\
\hline $\operatorname{Dmax}(\mathrm{cm})$ & 100,27 & 80,15 & 59,52 & 48,9 & 60,63 \\
\hline Amplitude (cm) & 90,27 & 69,74 & 49,21 & 38,9 & 50,60 \\
\hline Variância $\left(\mathrm{cm}^{2}\right)$ & 202,55 & 281,29 & 183,91 & 83,80 & 137,82 \\
\hline Desvio Padrão (cm) & 14,23 & 16,77 & 13,6 & 9,15 & 11,74 \\
\hline Assimetria & 1,44 & $-0,29$ & $-0,17$ & 1,16 & 0,85 \\
\hline Curtose & 1,87 & $-0,74$ & $-0,81$ & 0,87 & 0,51 \\
\hline
\end{tabular}

Dmin = DAP mínimo; Dmax = DAP máximo; e № = número de árvores avaliadas. 
A comunidade florestal foi afetada com o manejo, aumentando o número de árvores por hectare nas classes de menor diâmetro sem perder indivíduos nas classes maiores. Em 1993 a floresta apresentava 433 árvores por hectare, no ano de 2013 a floresta apresentou 507 árvores por hectare, evidenciando que ocorreu regeneração natural em fluxo contínuo e aumentou o número de árvores após o manejo. Árvores maiores na comunidade florestal ultrapassaram os $100 \mathrm{~cm}$ de diâmetro máximo, indicando que na execução do manejo foram preservados indivíduos em todas as classes de diâmetro e, em especial, as portas sementes. Porém, pode se verificar que houve redução de aproximadamente oito $\mathrm{cm}$ do DAP máximo do
C. fissilis, indicando que na execução do manejo essa espécie foi altamente explorada nas classes diamétricas maiores. A única espécie que aumento o diâmetro máximo em 2013 foi a $C$. xanthocarpa.

Na Tabela 2 estão apresentados os valores do teste de Kolmogorov-Smirnov e o ranqueamento do desempenho de todas as funções testadas para a floresta e para as espécies Araucaria angustifolia, Cedrela fissilis, Campomanesia xanthocarpa e Sebastiania commersoniana. Os resultados demonstraram que apenas a função Exponencial 2P apresentou aderência nos dois anos de mensuração para a comunidade florestal.

Tabela 2. Teste de Kolmogorov-Smirnov sobre as funções testadas para a distribuição diamétrica de um fragmento em ecótono de Floresta Ombrófila Mista e Floresta Estacional Decidual, e para quatro espécies selecionadas.

\begin{tabular}{|c|c|c|c|c|c|}
\hline \multirow{2}{*}{ Funções } & \multicolumn{2}{|c|}{ KS calculado } & \multicolumn{2}{|c|}{ Ranque } & \multirow{2}{*}{ Ranque total } \\
\hline & 1993 & 2013 & 1993 & 2013 & \\
\hline \multicolumn{6}{|c|}{ Floresta } \\
\hline Beta & $0,082^{\mathrm{ns}}$ & $0,156^{\text {ns }}$ & 4 & 5 & 9 \\
\hline Exponencial 2P & $0,037 * *$ & $0,033 * *$ & 1 & 1 & 2 \\
\hline Gama 3P & $0,072^{\text {ns }}$ & $0,064^{\mathrm{ns}}$ & 3 & 4 & 7 \\
\hline LogNormal 3P & $0,066^{\text {ns }}$ & $0,214^{\text {ns }}$ & 2 & 6 & 8 \\
\hline SB Johnson & $0,112^{\text {ns }}$ & $0,060^{\text {ns }}$ & 5 & 3 & 8 \\
\hline Weibull 3P & $0,141^{\text {ns }}$ & $0,055^{\text {ns }}$ & 6 & 2 & 8 \\
\hline \multicolumn{6}{|c|}{ Araucaria angustifolia (Bertol) Kuntze } \\
\hline Beta & $0,102^{*}$ & $0,068^{*}$ & 1 & 2 & 3 \\
\hline Exponencial 2P & $0,138^{*}$ & $0,240^{\text {ns }}$ & 2 & 6 & 8 \\
\hline Gama 3P & $0,171^{* *}$ & $0,075^{*}$ & 6 & 3 & 9 \\
\hline LogNormal 3P & $0,161^{* *}$ & $0,076^{*}$ & 5 & 4 & 9 \\
\hline SB Johnson & $0,143^{*}$ & $0,064^{*}$ & 4 & 1 & 5 \\
\hline Weibull 3P & $0,140 *$ & $0,077^{*}$ & 3 & 5 & 8 \\
\hline \multicolumn{6}{|c|}{ Cedrela fissilis Vell. } \\
\hline Beta & $0,122^{*}$ & $0,105^{*}$ & 6 & 5 & 11 \\
\hline Exponencial 2P & $0,098^{*}$ & $0,239 * *$ & 4 & 6 & 10 \\
\hline Gama 3P & $0,102^{*}$ & $0,094^{*}$ & 5 & 4 & 9 \\
\hline LogNormal 3P & $0,065^{*}$ & $0,094^{*}$ & 2 & 3 & 5 \\
\hline SB Johnson & $0,065^{*}$ & $0,067^{*}$ & 1 & 1 & 2 \\
\hline Weibull 3P & $0,091 *$ & $0,093^{*}$ & 3 & 2 & 5 \\
\hline \multicolumn{6}{|c|}{ Campomanesia xanthocarpa O. Berg } \\
\hline Beta & $0,113^{*}$ & $0,156 * *$ & 4 & 6 & 10 \\
\hline Exponencial 2P & $0,152 * *$ & $0,036^{*}$ & 6 & 2 & 8 \\
\hline Gama 3P & $0,131 * *$ & $0,050^{*}$ & 5 & 3 & 8 \\
\hline LogNormal 3P & $0,050 *$ & $0,090^{*}$ & 2 & 5 & 7 \\
\hline SB Johnson & $0,049 *$ & $0,030^{*}$ & 1 & 1 & 2 \\
\hline Weibull 3P & $0,108^{*}$ & $0,056^{*}$ & 3 & 4 & 7 \\
\hline \multicolumn{6}{|c|}{ Sebastiania commersoniana (Baill.) L.B. Sm. e Downs } \\
\hline Beta & $0,179 *$ & $0,160 *$ & 6 & 6 & 12 \\
\hline Exponencial 2P & $0,136^{*}$ & $0,088^{*}$ & 3 & 3 & 6 \\
\hline Gama 3P & $0,117^{*}$ & $0,105^{*}$ & 2 & 5 & 7 \\
\hline LogNormal 3P & $0,109 *$ & $0,070^{*}$ & 1 & 1 & 2 \\
\hline SB Johnson & $0,144^{*}$ & $0,072^{*}$ & 4 & 2 & 6 \\
\hline Weibull 3P & $0,177^{*}$ & $0,092^{*}$ & 5 & 4 & 9 \\
\hline
\end{tabular}


Com exceção da função exponencial 2P no ano de 2013 para A. angustifolia, todas as funções apresentaram aderência para as quatro espécies nos dois anos de avaliação. Para as espécies $C$. fissilis e $C$. xanthocarpa a função SB Johnson apresentou a melhor aderência do ajuste, já para a $A$. angustifolia foi a função Beta e para a $S$. commersoniana a função LogNormal 3P. Nas tabelas de 3 e 4 estão apresentados os parâmetros e os erros padrões $\left(\mathrm{S}_{\mathrm{yx}} \%\right)$ de todas as funções ajustadas para a comunidade florestal e as quatro espécies analisadas.
Por meio da análise gráfica das distribuições da comunidade florestal (Figura 1), verificou-se que ela, segue o padrão das florestas naturais, ou seja, apresentou a forma exponencial negativa ("J" invertido). Quando analisada as espécies separadamente, evidenciou-se que nem todas apresentam distribuição exponencial negativa (Figura 2), cabendo ressaltar que a espécie $C$. fissilis apresentou distribuição exponencial negativa em 1993 e distribuição unimodal em 2013.

Tabela 3. Parâmetros estimados das funções densidade de probabilidade ( $f d p s)$ para 1993 e 2013, em ecótono de Floresta Ombrófila Mista e Floresta Estacional Decidual, em Erval Seco, RS, Brasil.

\begin{tabular}{|c|c|c|c|c|c|c|c|c|c|c|}
\hline \multirow{2}{*}{ Função } & \multicolumn{9}{|c|}{ Coeficientes } & \multirow{2}{*}{$\mathrm{S}_{\mathrm{yx}} \%$} \\
\hline & $\alpha$ & $\beta$ & $\gamma$ & $\mu$ & $\lambda$ & $\delta$ & $\xi$ & $a$ & $\mathrm{~b}$ & \\
\hline \multicolumn{11}{|c|}{1993} \\
\hline Beta & 0,8158 & 3,7862 & - & - & - & - & - & 10,0 & 115,2 & 31,75 \\
\hline Exponencial 2P & - & - & 10,0 & - & 0,05955 & - & - & - & - & 22,73 \\
\hline Gama 3P & 0,8446 & 20,086 & 10,0 & - & - & - & - & - & - & 18,98 \\
\hline LogNormal 3P & 1,0001 & - & 8,4064 & 2,4931 & - & - & - & - & - & 27,30 \\
\hline SB Johnson & - & - & 1,2842 & - & 87,483 & 0,91683 & 5,5976 & - & - & 43,17 \\
\hline Weibull 3P & 0,76241 & 16,256 & 10,0 & - & - & - & - & - & - & 22,95 \\
\hline \multicolumn{11}{|c|}{2013} \\
\hline Beta & 0,67671 & 3,6406 & - & - & - & - & - & 10,0 & 120,32 & 47,91 \\
\hline Exponencial 2P & - & - & 10,0 & & 0,07068 & - & - & - & - & 19,21 \\
\hline Gama 3P & 0,8303 & 17,234 & 10,0 & - & - & - & - & - & - & 18,30 \\
\hline LogNormal 3P & 1,0872 & - & 9,2544 & 2,1997 & - & - & - & - & - & 76,22 \\
\hline SB Johnson & - & - & 1,5099 & - & 85,139 & 0,79713 & 8,3671 & - & - & 21,74 \\
\hline
\end{tabular}

$a, b, \alpha, b, \gamma, \mu, \lambda, \delta, \xi=$ parâmetros dos modelos; e $\mathrm{S}_{\mathrm{yx}} \%$ = erro padrão da estimativa em percentagem.
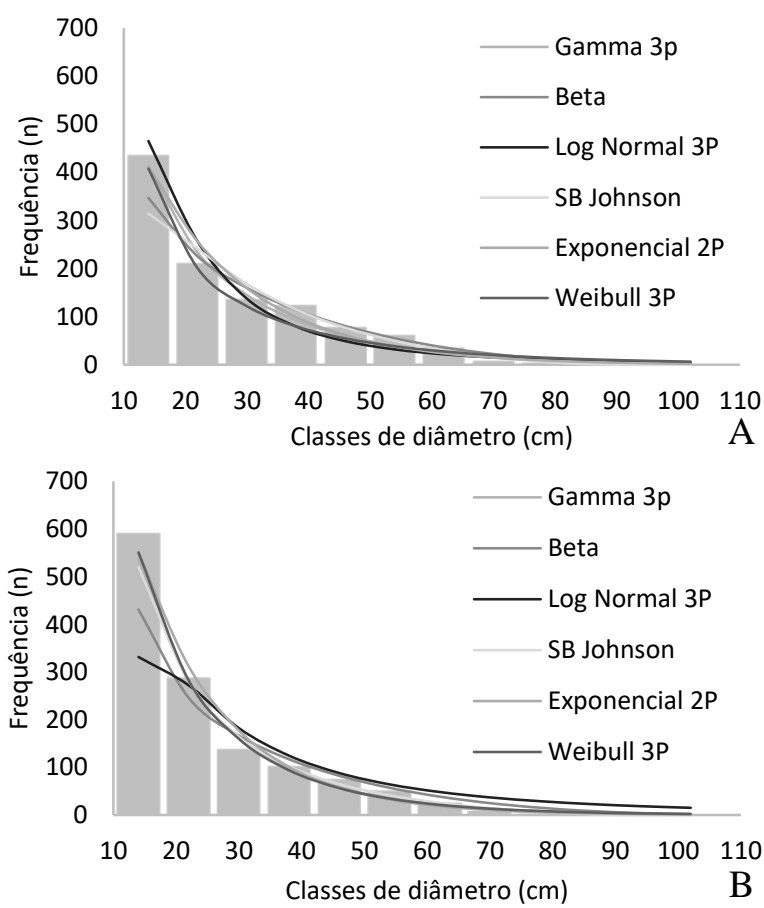

Figura 1. Distribuições diamétrica nos períodos 1993 (A) e 2013 (B), em ecótono de Floresta Ombrófila Mista e Floresta Estacional Decidual, em Erval Seco, RS, Brasil.
As distribuições diamétricas são caracterizadas como assimétricas quando a média, mediana e moda incidem em diferentes posições da curva, cujos deslocamentos podem ocorrer para a direita ou esquerda (MACHADO et al., 2006; SANTOS et al., 2016). Para a comunidade florestal e para as espécies $C$. xanthocarpa, S. commersoniana, nos anos de 1993 e 2013, e C. fissilis, em 1993 (Figura 2), a assimetria foi positiva, sendo, essa considerada moderada quando os coeficientes (em módulo) variam de 0,15 a 1,00 e, forte quando maior que um (MACHADO et al., 2006).

Para a A. angustifolia, nos anos de 1993 e 2013, e C. fissilis em 2013, a assimetria foi negativa, podendo ser indicativo de que a regeneração não ocorre de maneira contínua (EBLING e PÉLLICO NETTO, 2015). Para verificar a influência do manejo florestal em regime sustentado sobre o fragmento florestal e nas quatro espécies em estudo, selecionou-se a " $f d p$ " que apresentou a melhor aderência. Para a floresta com um todo foi selecionada a distribuição Exponencial 2P.

Para as espécies A. angustifolia, C. fissilis e C. xanthocarpa e $S$. commersoniana foram selecionadas as distribuições de Beta, SB de Johnson, SB de Johnson, e Log- 

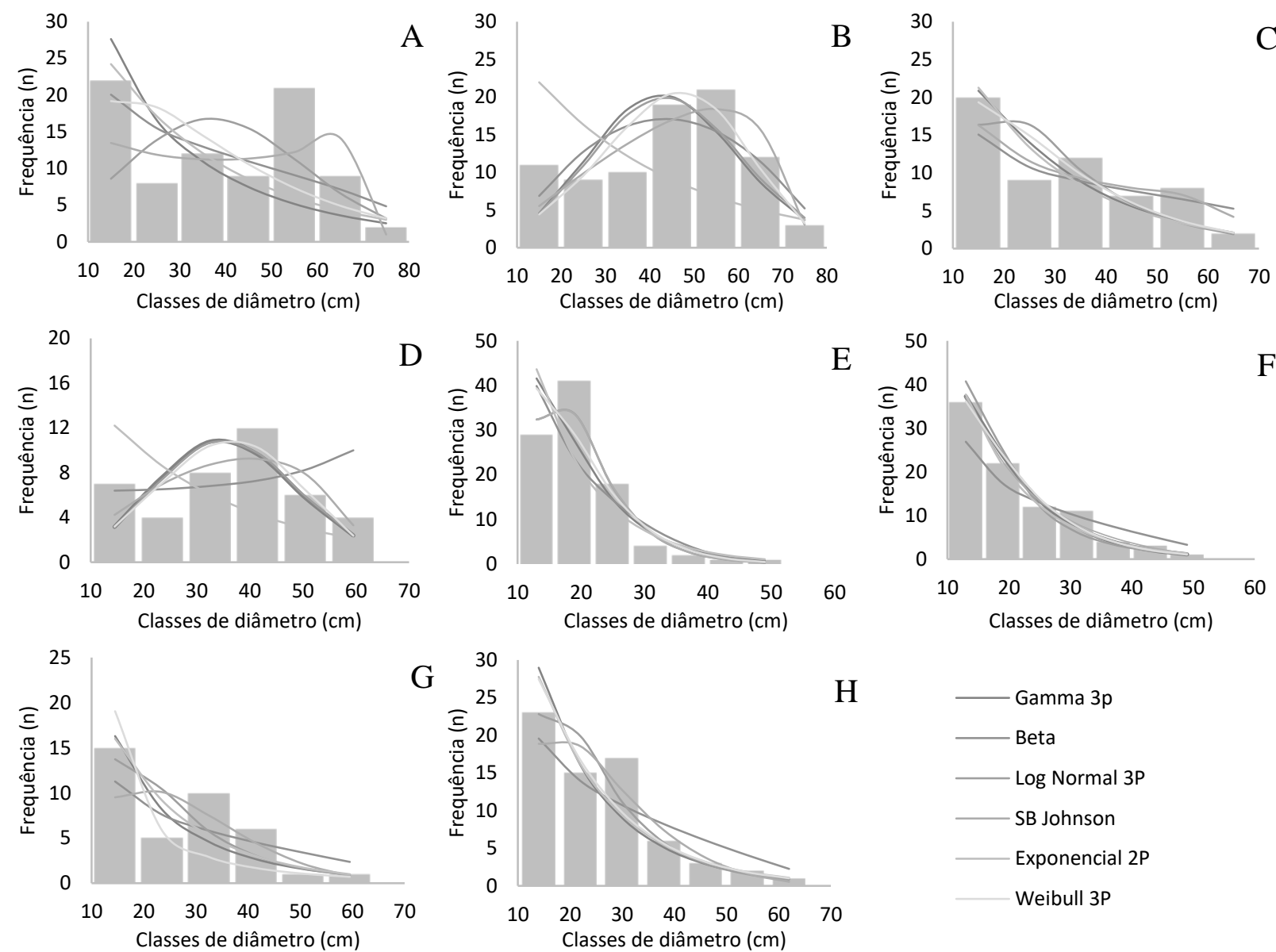

$\mathrm{H}$

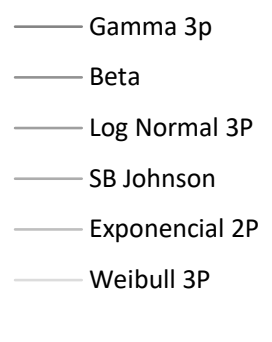

Figura 2. Distribuições diamétrica de Araucaria angustifolia nos anos 1993 (A) e 2013 (B), Cedrela fissilis nos anos 1993 (C) e 2013 (D), Campomanesia xanthocarpa nos anos 1993 (E) e 2013 (F) e Sebastiania commersoniana nos anos 1993 (G) e $2013(\mathrm{H})$, em ecótono de Floresta Ombrófila Mista e Floresta Estacional Decidual, em Erval Seco, RS, Brasil.

Normal 3P, respectivamente. Com a análise individual de cada espécie foi possível observar que o manejo provocou mudanças nas distribuições diamétricas das espécies $A$. angustifolia, $C$. fissilis e $C$. xanthocarpa (Figura 3). Para as espécies $A$. angustifolia e $C$. fissilis a distribuição diamétrica em 1993 foi exponencial negativa e com a execução do manejo a distribuição diamétrica em 2013 se tornou unimodal, considerando que para $C$. fissilis a frequência diminuiu em todas as classes de diâmetros. O teste de QuiQuadrado demonstrou não haver diferença significativa entre as curvas ajustadas para a comunidade e as espécies estudadas (Figura 3).

A regeneração em fluxo descontínuo pode ser uma caraterística intrínseca das espécies, as quais apresentam ciclos de regeneração (CYSNEIROS et al., 2017), ou necessitam da incidência de distúrbios para que ela ocorra. No presente trabalho a baixa regeneração nas classes iniciais da distribuição diamétrica para a A. angustifolia e C. fissilis foi reflexo do manejo, pois essas espécies são classificadas como não pioneiras (MACHADO et al., 2017).
A redução de indivíduos nas classes iniciais da distribuição de $A$. angustifolia também foi observada por Silveira et al. (2018) em trabalho realizado em fragmento não manejado em Lages, Santa Catarina, BR, com inventários realizados em 2012 e 2016. Dessa forma, o período de 20 anos após o manejo pode ser pouco para se obter recrutamento de $A$. angustifolia nas classes iniciais de diâmetro. Assim, estudos com períodos maiores pós manejo devem ser realizados para identificar os coortes da A. angustifolia, como encontrado por Ebling e Péllico Netto (2015).

Silveira et al. (2018) concluíram que o manejo florestal sustentável dessa população propiciará mais espaço para as demais árvores, melhorando seu desenvolvimento, uma vez que reduziria a competição por espaço, luz e nutrientes. No presente estudo, com período de 20 anos entre os inventários, verificou-se redução no número de árvores nas classes com menos $50 \mathrm{~cm}$ para $A$. angustifolia (Figura 2A e 2B). 
Tabela 4. Parâmetros estimados das funções densidade de probabilidade (fdps) para 1993 e 2013 das espécies Araucaria angustifolia, Cedrela fissilis, Campomanesia xanthocarpa e Sebastiania commersoniana, em ecótono de Floresta Ombrófila Mista e Floresta Estacional Decidual, em Erval Seco, RS, Brasil.

\begin{tabular}{|c|c|c|c|c|c|c|c|c|c|c|}
\hline \multirow{2}{*}{ Função } & \multicolumn{9}{|c|}{ Coeficientes } & \multirow{2}{*}{$\mathrm{S}_{y x} \%$} \\
\hline & $\alpha$ & $\beta$ & $\gamma$ & $\mu$ & $\lambda$ & $\delta$ & $\xi$ & a & $b$ & \\
\hline \multicolumn{11}{|c|}{ Araucaria angustifolia } \\
\hline \multicolumn{11}{|c|}{1993} \\
\hline Beta & 0,8352 & 1,5004 & - & - & - & - & - & 10,0 & 84,45 & 50,83 \\
\hline Exponencial 2P & - & - & 10,0 & - & 0,03471 & - & - & - & - & 63,06 \\
\hline Gama 3P & 0,8316 & 30,607 & 10,0 & - & - & - & - & - & - & 67,88 \\
\hline LogNormal 3P & 0,0648 & - & $-266,73$ & 5,7201 & - & - & - & - & - & 67,09 \\
\hline SB Johnson & - & - & $-0,0038$ & - & 67,326 & 0,56922 & 5,0779 & - & - & 48,47 \\
\hline Weibull 3P & 1,2518 & 31,288 & 9,3813 & - & - & - & - & - & - & 62,35 \\
\hline \multicolumn{11}{|c|}{2013} \\
\hline Beta & 2,0434 & 2,0762 & - & - & - & - & - & 6,18 & 82,07 & 35,08 \\
\hline Exponencial 2P & - & - & 10,409 & & 0,02959 & - & - & - & - & 77,47 \\
\hline Gama 3P & 114,43 & 1,5679 & $-135,53$ & - & - & - & - & - & - & 42,73 \\
\hline LogNormal 3P & 0,04246 & - & $-356,99$ & 5,9939 & - & - & - & - & - & 39,93 \\
\hline SB Johnson & - & - & $-0,40845$ & - & 81,945 & 0,99338 & $-3,6552$ & - & - & 28,05 \\
\hline Weibull 3P & 4,9878 & 77,199 & $-26,513$ & - & - & - & - & - & - & 32,25 \\
\hline \multicolumn{11}{|c|}{ Cedrela fissilis } \\
\hline \multicolumn{11}{|c|}{1993} \\
\hline Beta & 0,73049 & 1,221 & - & - & - & - & - & 10,0 & 74,58 & 32,75 \\
\hline Exponencial 2P & - & - & 10,0 & - & 0,04622 & - & - & - & - & 35,27 \\
\hline Gama 3P & 1,1028 & 19,665 & 9,9496 & - & - & - & - & - & - & 35,52 \\
\hline LogNormal 3P & 0,60633 & - & 1,9423 & 3,222 & - & - & - & - & - & 44,62 \\
\hline SB Johnson & - & - & 0,38246 & - & 58,912 & 0,60779 & 8,3598 & - & - & 27,02 \\
\hline Weibull 3P & 1,1706 & 22,847 & 9,8708 & - & - & - & - & - & - & 35,87 \\
\hline \multicolumn{11}{|c|}{2013} \\
\hline Beta & 0,98072 & 0,1419 & - & - & - & - & - & 10,309 & 59,524 & 55,28 \\
\hline Exponencial 2P & - & - & 10,313 & - & 0,03895 & - & - & - & - & 73,00 \\
\hline Gama 3P & 102,23 & 1,3395 & $-100,97$ & - & - & - & - & - & - & 43,31 \\
\hline LogNormal 3P & 0,04622 & - & $-257,67$ & 5,6815 & - & - & - & - & - & 42,10 \\
\hline SB Johnson & - & - & $-0,23716$ & - & 66,135 & 1,0114 & $-0,2837$ & - & - & 35,07 \\
\hline Weibull 3P & 3,6157 & 47,072 & $-6,3299$ & - & - & - & - & - & - & 39,18 \\
\hline \multicolumn{11}{|c|}{ Campomanesia xanthocarpa } \\
\hline \multicolumn{11}{|c|}{1993} \\
\hline Beta & 0,83249 & 3,1389 & - & - & - & - & - & 10,0 & 53,585 & 63,74 \\
\hline Exponencial 2P & - & - & 10,0 & - & 0,1033 & - & - & - & - & 70,54 \\
\hline Gama 3P & 1,4366 & 7,0196 & 9,5957 & - & - & - & - & - & - & 56,71 \\
\hline LogNormal 3P & 0,40888 & - & 2,3897 & 2,7674 & - & - & - & - & - & 25,97 \\
\hline SB Johnson & - & - & 6,6695 & - & 319,86 & 2,2311 & 3,0023 & - & - & 26,24 \\
\hline Weibull 3P & 1,3009 & 10,77 & 9,6673 & - & - & - & - & - & - & 48,72 \\
\hline \multicolumn{11}{|c|}{2013} \\
\hline Beta & 0,71497 & 1,8851 & - & - & - & - & - & 10,0 & 58,68 & 36,43 \\
\hline Exponencial 2P & - & - & 10,0 & - & 0,09349 & - & - & - & - & 14,28 \\
\hline Gama 3P & 1,205 & 8,9107 & 9,9596 & - & - & - & - & - & - & 14,72 \\
\hline LogNormal 3P & 0,80769 & - & 8,6478 & 2,1907 & - & - & - & - & - & 24,13 \\
\hline SB Johnson & - & - & 1,2906 & - & 50,36 & 0,8294 & 9,2718 & - & - & 11,17 \\
\hline Weibull 3P & 1,1332 & 11,204 & 9,9746 & - & - & - & - & - & - & 14,33 \\
\hline
\end{tabular}




\begin{tabular}{|c|c|c|c|c|c|c|c|c|c|c|}
\hline \multicolumn{11}{|c|}{ Sebastiania commersoniana } \\
\hline \multicolumn{11}{|c|}{1993} \\
\hline Beta & 0,74744 & 1,7471 & - & - & - & - & - & 10,0 & 76,776 & 48,90 \\
\hline Exponencial 2P & - & - & 10,0 & - & 0,06183 & - & - & - & - & 50,11 \\
\hline Gama 3P & 0,8049 & 18,862 & 10,0 & - & - & - & - & - & - & 51,15 \\
\hline LogNormal 3P & 0,65619 & - & 3,658 & 2,9196 & - & - & - & - & - & 53,91 \\
\hline SB Johnson & - & - & 1,3413 & - & 98,661 & 1,462 & $-3,7268$ & - & - & 57,37 \\
\hline Weibull 3P & 0,34972 & 8,5241 & 10,0 & - & - & - & - & - & - & 65,56 \\
\hline \multicolumn{11}{|c|}{2013} \\
\hline Beta & 0,82644 & 2,0105 & - & - & - & - & - & 10,027 & 72,766 & 36,22 \\
\hline Exponencial 2P & - & - & 10,027 & - & 0,06783 & - & - & - & - & 38,73 \\
\hline Gama 3P & 0,97196 & 14,272 & 10,027 & - & - & - & - & - & - & 42,93 \\
\hline LogNormal 3P & 0,5934 & - & 3,8907 & 2,8739 & - & - & - & - & - & 32,63 \\
\hline SB Johnson & - & - & 1,6157 & - & 88,553 & 1,3176 & 2,5756 & - & - & 30,37 \\
\hline Weibull 3P & 1,0401 & 14,949 & 10,015 & - & - & - & - & - & - & 37,07 \\
\hline
\end{tabular}

$a, b, \alpha, b, \gamma, \mu, \lambda, \delta, \xi=$ parâmetros dos modelos; e $\mathrm{S}_{\mathrm{yx}} \%$ = erro padrão da estimativa em percentagem.
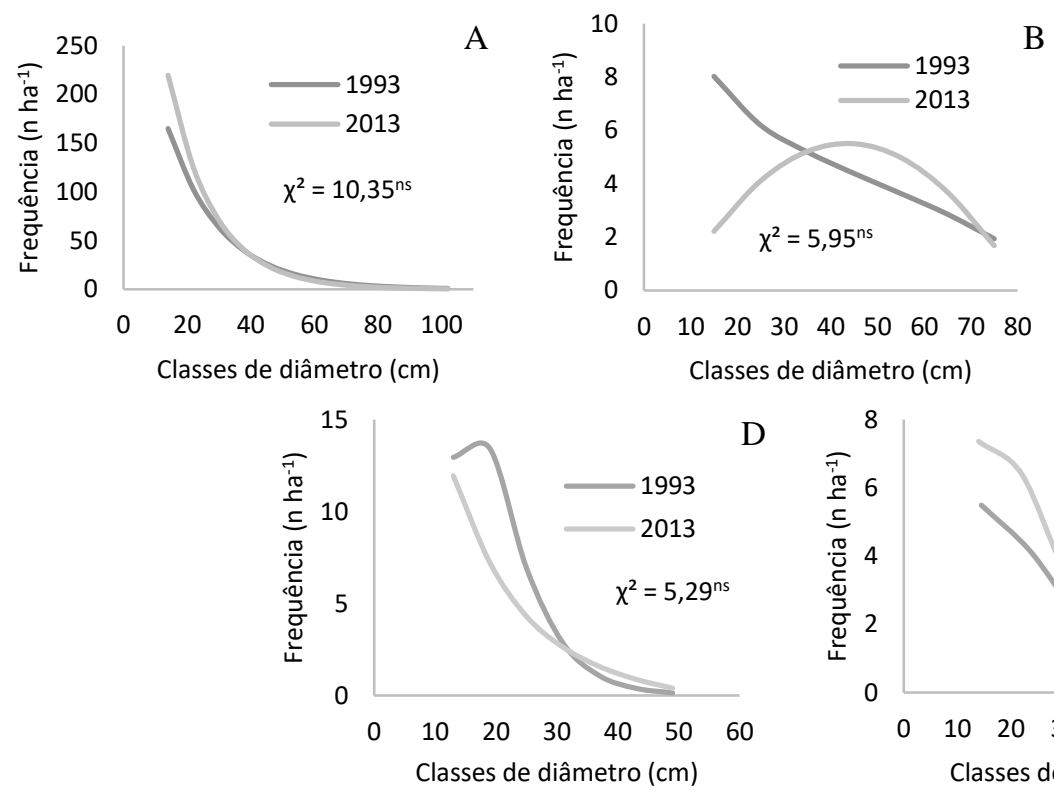

D

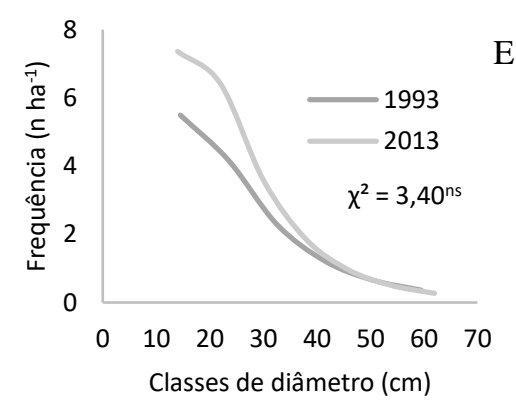

Figura 3. Distribuições diamétrica nos anos 1993 e 2013 em um fragmento de ecótono em Floresta Ombrófila Mista e Floresta Estacional Decidual (A) e de Araucaria angustifolia (B), Cedrela fissilis (C), Campomanesia xanthocarpa (D), Sebastiania commersoniana (E), em Erval Seco, RS, Brasil.

No ano de 2013 foi possível perceber visualmente que a regeneração da $A$. angustifolia esteve presente, porém a maioria dessas árvores não atingiram o diâmetro mínimo de inclusão (DAP > $10 \mathrm{~cm}$ ). Esses fatos podem estar associados a exploração de forma descontrolada da colheita do pinhão nos primeiros anos após o manejo, pois, próximo ao fragmento, existe uma comunidade extrativista que sobrevive da exploração da erva-mate e do pinhão.

A melhor aderência da função Exponencial $2 \mathrm{P}$ já era esperada, pois florestas naturais apresentam a distribuição diamétrica em forma de "J" invertido, como já relatado por outros pesquisadores em florestas tropicais (SANTOS et al.,
2016; CYSNEIROS et al., 2017) e florestas subtropicais (TÉO et al., 2015; MACHADO et al., 2017; LONGHI et al., 2017). Outro trabalho realizado em floresta Ombrófila Mista subtropicais evidenciaram a distribuição de SB de Johnson (TÉO et al., 2015) com as melhores aderências aos dados reais. Em estudo realizado em floresta Ombrófila Densa tropical, a distribuição SB de Johnson também apresentou o melhor ajuste (CYSNEIROS et al., 2017). Em geral, na análise individual das espécies, as distribuições Beta, LogNormal 3P, SB de Johnson e Weibull 3P apresentaram boa aderência aos dados diamétricos de todas as espécies estudadas e para os dois anos de avalição, demonstrando que essas $f d p s$ desempenharam boa flexibilidade a 
diferentes situações.

Das quatro espécies analisadas nos dois anos de avaliação, apenas C. xanthocarpa, S. commersoniana apresentaram distribuição em "J" invertido em 2013 e em 1993 a C. xanthocarpa não apresentava essa distribuição, devido à ausência de indivíduos em uma das duas primeiras classes. Esse fato pode ser explicado pela ocorrência de alguma perturbação natural que afetou o estabelecimento e desenvolvimento dessas espécies e provocou padrões irregulares na distribuição (ALVES; METZGER, 2006; SANTOS et al., 2016). Para as espécies $C$. xanthocarpa, S. commersoniana o manejo em regime sustentado propiciou o restabelecimento da regeneração natural em fluxo contínuo.

\section{CONCLUSÕES}

O manejo florestal em regime sustentado propicia benefícios para o estabelecimento da regeneração de algumas espécies, como para Campomanesia xanthocarpa que retomou a distribuição de "J" invertido no ano de 2013 e Sebastiania commersoniana que aumento o número de árvores nas classes iniciais de DAP.

Com a execução do manejo florestal as espécies Araucaria angustifolia e Cedrela fissilis não apresentam distribuição de "J" invertido no ano de 2013. Um novo manejo deve ser realizado na floresta, tomando medidas preventivas para propiciar o recrutamento da regeneração nas classes iniciais dessas duas espécies.

Para a floresta o manejo apresenta efeitos positivos, pois a distribuição é exponencial negativa no ano de 2013, com aumento do número de árvores nas classes iniciais.

\section{REFERÊNCIAS}

ALVARES, C.A. et al. Köppen's climate classification map for Brazil. Meteorologische Zeitschrift, v.22, n.6, p.711-728, 2013.

ALVES, L.F.; METZGER, J.P. A regeneração florestal em áreas de floresta secundária na Reserva Florestal do Morro Grande, Cotia, SP. Biota Neotropical, v.6, p.1-26, 2006.

BINOTI, D.H.B. et al. Funções densidade de probabilidade para descrição da distribuição diamétrica de povoamentos de Acacia mangium. Revista Brasileira de Ciências Agrárias, v.9, n.4, p.583589, 2014

CYSNEIROS, V.C. et al. Distribuição diamétrica de espécies da Floresta Ombrófila Densa no Sul do Estado do Rio de Janeiro. Pesquisa Florestal Brasileira, v.37, n.89, p.1-10, 2017.

EBLING, A.A.; PÉLLICO NETTO, S. Modelagem de ocorrência de coortes na estrutura diamétrica da Araucaria angustifolia (Bertol.) Kuntze. Cerne, v.21, n.2, p.251-257, 2015.
HESS, A.F. et al. Proposta de manejo de Araucaria angustifolia utilizando o quociente de Liocourt e análise de incremento, em propriedade rural no Município de Lages, SC. Pesquisa Florestal Brasileira, v.30, n.64, p.337-345, 2010.

IBGE. Manual técnico da vegetação brasileira. Rio de Janeiro. IBGE. 2012.

LOETSCH, F. et al. Forest inventory. München: BLV Verlagsgesellschaft, 1973.

LONGHI, R.V. et al. Projeção da distribuição diamétrica em floresta com Araucária explorada seletivamente no Sul do Brasil. Revista Brasileira de Ciências Agrárias, v.12, n.2, p.210-219, 2017.

MACHADO, S.A. et al. Dinâmica da distribuição diamétrica de bracatingais na região metropolitana de Curitiba. Revista Árvore, v.30, n.5, p.759-768, 2006.

MACHADO, S. A. et al. Projeção da estrutura diamétrica de grupos ecológicos em uma Floresta Ombrófila Mista. Floresta e Ambiente, v.24, e20160012, 2017.

MACHADO, S.A. et al. Distribution of total height, transverse area and individual volume for Araucaria angustifolia (Bert.) O. Kuntze. Cerne, v.16, n.1, p.12-21, 2010.

MACHADO, S.A. et al. Comparação dos parâmetros fitossociológicos entre três estratos de um fragmento de floresta ombrófila mista. Cerne, v.19, n.3, p.365-372, 2013.

ROSSATO, M.S. Os climas do Rio Grande do Sul: Tendências e tipologias. Os climas do Sul: Em tempos de mudanças climáticas globais: Paco Editorial, p.217-271, 2014.

SANTOS, R.O. et al. Distribuição diamétrica de uma comunidade arbórea na Floresta Estadual do Amapá, Brasil. Biota Amazônia, v.6, n.2, p.24-31, 2016.

SANTOS, H.G. et al. Sistema brasileiro de classificação de solos. 3.ed. Brasília: Embrapa, 2013.

SCOLFORO, J.R.S. Biometria florestal: modelos de crescimento e produção florestal. Lavras: UFLA/FAEPE, 2006.

SILVEIRA, A.C. et al. Management of Brazilian pine (Araucaria angustifolia (Bertol) Kuntze) based on the Liocourt model in a mixed ombrophilous forest in Southern Brazil. Australian Journal of Crop Science, v.12, n.2, p.311-317, 2018.

STURGES, $\mathrm{H}$. The choice of a class interval. Journal of the American Statistical Association, v.21, n.153, p.65-66, 1926.

TÉO, S.J. et al. Modelagem da distribuição diamétrica de um fragmento de floresta ombrófila mista em Lebon Régis, SC. Floresta, v.45, n.2, p.337-348, 2015.

VENDRUSCOLO, R. Dinâmica fitossociológica e de crescimento de um fragmento florestal manejado em 1993, no noroeste do RS. 2015. 77p. (Dissertação de mestrado). 